Follow-up occurred in 122(76\%), clients including 26/28 with F3-4 fibrosis. Thirteen clients were able to access clinical trials of HCV treatment.

Conclusion This study demonstrates the utility of delivering a fibroscan service by a health facility that focusses on STIs, HIV and hepatitis. Uptake and retention in care was achieved for this marginalised population. The Hepatitis C strategies' focus on primary health care and sexual health services for HCV care and treatment in an era of interferon-free therapy appears feasible.

Disclosure of interest statement No conflicts of interest to declare.

\section{P13.04 "I DO FEEL LIKE A SCIENTIST AT TIME YEAH..." ACCEPTABILITY OF POINT-OF-CARE TESTING FOR CHLAMYDIA AND GONORRHOEA TO HEALTH SERVICE PROVIDERS IN REMOTE PRIMARY CARE}

1,2L Natoli*, ${ }^{3} \mathrm{~T}$ Coburn*, 'L Maher, ${ }^{4} \mathrm{M}$ Shephard, ${ }^{5} \mathrm{~B}$ Hengel, 'S Badman, ${ }^{1} \mathrm{~L}$ Causer, ${ }^{6} \mathrm{~A}$ Tangey, ${ }^{1} \mathrm{~J}$ Kaldor, ${ }^{1,7} \mathrm{~J}$ Ward, ${ }^{2} \mathrm{D}$ Anderson, ${ }^{1} \mathrm{R}$ Guy, on behalf of the TTANGO Investigators. 'Kirby Institute, University of New South Wales, Sydney, Australia; ${ }^{2}$ The Burnet Institute, Melbourne, Australia; ${ }^{3}$ Queensland Aboriginal and Islander Health Council; ${ }^{4}$ Flinders University, Adelaide, Australia; ${ }^{5}$ Apunipima Cape York Health Council, Cairns, Australia; ${ }^{6}$ Ngaanyatjarra Health Service, Alice Springs, Australia; ${ }^{7}$ South Australian Health and Medical Research Institute, Adelaide, Australia

\subsection{6/sextrans-2015-052270.502}

Introduction The GeneXpert test system is a molecular test used to diagnose Chlamydia trachomatis and Neisseria gonorrhoeae at the point-of-care (POC). It is being used in remote Aboriginal health services in Australia as part of the TTANGO (Test, Treat, ANd GO) Trial.

Methods In 2014 we interviewed 15 Aboriginal health workers/ practitioners and nurses from 6 health services participating in TTANGO. Most were female (53\%), 70\% had worked $>5$ years in the remote sector and $40 \%$ were Aboriginal. We explored factors known to influence POC test acceptability including perceived ease of use and usefulness, and staff attitudes- which are all mediated by a range of barriers and enablers to POC test use. Results Most staff found the GeneXpert both easy to use and useful in their setting. They indicated that POC testing has improved STI management, resulting in more timely and targeted treatment, earlier commencement of partner notification, and reduced time and effort associated with client recall. Staff expressed confidence in POC test results and in treating patients on this basis. They reported greater job satisfaction- feeling more in control of STI testing and patient health. Access to the GeneXpert appeared to legitimise or create an entry point to discussing STIs with clients, particularly for Aboriginal health workers. As most clients opted to return for test results (after $90 \mathrm{~min}$ ) POC testing did not impact negatively on client flow. Managing positive test results in a shorter time frame was sometimes challenging. Manual documentation of results was considered to be onerous by some, who suggested that enhanced connectivity between the GeneXpert and patient management system could assist.

Conclusion Participants identified the potential for the GeneXpert to strengthen STI control in remote communities. Test acceptability was high, although some challenges remain and will inform future scale up/translation of POC testing in this setting. Disclosure of interest statement No conflicts of interest declared. No financial support was received by Cepheid. Cepheid has provided GeneXpert devices on loan for the duration of TTANGO and test cartridges at a reduced rate.
P13.05 PREVENTION OF MOTHER-TO-CHILD TRANSMISSION OF SYPHILIS AND HIV IN CHINA: WHAT DRIVES POLITICAL PRIORITISATION AND WHAT CAN THIS TELL US ABOUT PROMOTING DUAL CONTROL?

${ }^{1} \mathrm{D}$ Wu*, 'S Hawkes, ${ }^{2} \mathrm{~K}$ Buse. ${ }^{1}$ Institute for Global Health, University College London, UK; ${ }^{2}$ The Joint United Nations Programme on HIVIAIDS (UNAIDS), Geneva, Switzerland

\subsection{6/sextrans-2015-052270.503}

Introduction Despite a large and growing burden of mother-tochild transmission (MTCT) of syphilis in China over the past 20 years, the issue received far less attention and fewer resources than prevention of MTCT (PMTCT) of HIV, which has a substantially lower burden. China's Ministry of Health issued the first national plan for syphilis control in 2010, aiming to integrate PMTCT of syphilis and HIV. Our study aimed to identify: 1) why PMTCT of syphilis had a lower political/resource priority than PMTCT of HIV before 2010; and 2) what actions would improve the prospects of successful implementation of dual PMTCT.

Methods We undertook a comparative policy analysis, based on informant interviews, documentation review, and nonparticipant observation of relevant meetings/trainings, to investigate prioritysetting prior to 2010. We used a nine-factor framework developed by Shiffman et al. which assesses political prioritisation across three categories: transnational influence; domestic advocacy; and national political environment.

Results We identified several factors contributing to the lower priority accorded to PMTCT of syphilis: 1) relative neglect at a global level; 2) dearth of international financial and technical support; 3) poorly unified national policy community with weak accountability mechanisms; 4) insufficient understanding of the epidemic and policy options; and 5) a prevailing negative framing of syphilis that resulted in significant stigmatisation.

Conclusion The goal of dual PMTCT of syphilis and HIV will only be achieved when equal priority is accorded to both infections. This will require stronger cohesion and leadership from the syphilis policy community. The community will also need to reframe the issue so as to overcome stigmatisation against those affected by the illness, organise focusing events to attract political attention, and work more closely with the HIV policy community in order to enhance the recognition of the need to control syphilis on both the national and sub-national agendas. Disclosure of interest statement This study was funded by the Department of Reproductive Health and Research, World Health Organization during the period from September 2011 to August 2012. The views expressed are not necessarily those of UNAIDS.

\section{P13.06 KNOWLEDGE TRANSLATION: DEVELOPMENT OF A SEXUAL HEALTH CLINICAL AUDIT TOOL TO ENHANCE ADHERENCE TO EVIDENCE-BASED GUIDELINES}

${ }^{1} \mathrm{~B}$ Nattabi*, ${ }^{2} \mathrm{~S}$ Kanai, ${ }^{2} \mathrm{~S}$ Ferguson-Hill, ${ }^{2} \mathrm{D}$ Mosca, ${ }^{3} \mathrm{M}$ Murphy, ${ }^{3} \mathrm{R}$ Bailie. ${ }^{1}$ Western Australian Centre for Rural Health, University of Western Australia; ${ }^{2}$ National Centre for Quality Improvement in Indigenous Primary Health Care (One21seventy); ${ }^{3}$ Menzies School of Health Research, Brisbane

\subsection{6/sextrans-2015-052270.504}

Introduction Sexually transmitted infections remain a significant public health issue for Indigenous Australians. Reasons for the high burden of disease include lack of access to quality care particularly in rural and remote Australia. Commissioned by the 
ABCD National Research Partnership, this project aimed to develop an audit tool to be used within a continuous quality improvement approach to enhance adherence to best-practice guidelines and improve the quality of Indigenous primary sexual health services.

Methods The process of development of the tool involved engagement of a range of stakeholders including clinical experts, quality improvement practitioners and researchers; identification and review of best practice guidelines; development of key indicators that reflect quality of care; generation of audit items and questions; and construction of the tool, protocol and report. The tool was piloted in Western Australia, Northern Territory, Queensland and South Australia.

Results The sexual health tool includes indicators that cover the basic elements of sexual health care including risk assessment, investigations, treatment, contact tracing and follow up. The protocol guides the use of the tool and the tailored report assists in identification of gaps, goal setting and planning of actions for improvement. Important elements of tool development are broad end user engagement, multidisciplinary and multi-jurisdictional consultation, effective leadership, sufficient resources and consensus building around selection of key elements of sexual health care.

Conclusion The tool, which reflects the best practice for Indigenous primary sexual healthcare, is now available to Indigenous primary health care services through the National Centre for Quality Improvement in Indigenous Primary Health Care (One21seventy). Used in conjunction with the systems assessment tool, the tool will be used to identify evidence-practice gaps, determine systems-related facilitators and barriers to quality care enhance the quality of sexual health care delivered to, and ultimately reduce the burden of STIs among, Indigenous Australians.

Disclosure of interest statement Barbara Nattabi is supported by an NHMRC Early Career Research Fellowship \#1072777. Development of the tool was supported by the Lowitja Institute. No pharmaceutical grants were received in the development of this study.

\section{P13.07 WHICH ELEMENTS OF A NOVEL SELF-DIRECTED RAPID ASYMPTOMATIC SEXUALLY TRANSMITTED INFECTION SCREENING SERVICE ARE MOST IMPORTANT TO USERS?}

L Chislett*, J Clarke. Dean Street Express, Chelsea and Westminster Hospital NHS Foundation Trust London, UK

\subsection{6/sextrans-2015-052270.505}

Introduction In Feb 2014 we launched an innovative walk in asymptomatic screening service targeting higher risk groups in central London UK. Users self register and record their own sexual history using a touch screen which triggers automated test ordering. Users then are self-directed to take their own samples using video instructions. HIV point of care testing gives $60 \mathrm{~s}$ results. The use of on site Cepheid ${ }^{\circledR}$ GeneXpert allows samples to be processed within 90 min with results delivered by automated results management. Within 6 months the service was attracting over 6000 attendances per month. The aim of this survey was to ascertain the elements of the service that were most attractive to users.

Methods Prospective survey of sequential attendees.
Results 78\% identified as Gay/Bisexual, 20\% Heterosexual, 2\% Lesbian.

All respondents said that they would recommend the Dean Street Express model to a friend. Use of touch screen. Really Easy $78 \%$, Easy 22\%, OK 0\%, Hard 0\%, Very Hard 0\%. Selftaken samples. Really Easy 66\%, Easy 26\%, OK 8\%, Hard 0\%, Very Hard $0 \%$.

Percentage indicating important:

\begin{tabular}{ll}
\hline Walk in service & $96 \%$ \\
Rapid results & $76 \%$ \\
Location & $64 \%$ \\
Self taken swabs & $62 \%$ \\
Staff & $62 \%$ \\
Pleasant environment & $48 \%$ \\
\hline
\end{tabular}

Conclusion The use of touch screens, self sampling and rapid results through the use of on site diagnostics are highly acceptable to service users.

Disclosure of interest statement Leigh Chislett's travel and accommodation to this conference were sponsored by Cepheid.

\section{P13.08 ACT TESTING MONTH: PROMOTING TESTING AND INCREASING CROSS SECTOR COLLABORATION}

${ }^{1} \mathrm{M}$ Todkill*, ${ }^{1,2} \mathrm{~A}$ Tyson, ${ }^{3} \mathrm{P}$ Habel, ${ }^{4} \mathrm{P}$ Moss, ${ }^{4} \mathrm{~K}$ Rossteuscher, ${ }^{5} \mathrm{~J}$ Didlick, ${ }^{6} \mathrm{~S}$ Crawford, ${ }^{7} \mathrm{H}$ Freeman, ${ }^{1,2} \mathrm{SJ}$ Martin. ${ }^{1}$ Canberra Sexual Health Centre; ${ }^{2}$ Australian National University Medical School; ${ }^{3}$ ACT Medicare Local; ${ }^{4}$ AIDS Action Council of ACT; ${ }^{5}$ Hepatitis ACT; ${ }^{6}$ Canberra Alliance for Harm Minimisation and Advocacy; ${ }^{7}$ Sexual Health \& Family Planning ACT

\subsection{6/sextrans-2015-052270.506}

Background Prompted by an increase in HIV notifications, community support after AIDS 2014 and the success of NSW's inaugural HIV Testing Week, the Australian Capital Territory (ACT) held its first Testing Month in November 2014 to promote initial and ongoing testing for HIV, STI and hepatitis in priority populations.

Methods A stakeholder group collaborated to promote testing via social and mainstream media, offer targeted outreach testing and provide workplace-based GP education focussing on local epidemiology, national testing guidelines and participant perceptions of barriers to testing. Digital and print media was used to promote the Ending HIV campaign, Time to Test and Testing Month. ACT Testing Month was launched at a gay community event on 1 November and ran until World AIDS Day, 1 December 2014.

Results Social marketing focussed on testing with links to testing sites. Local media ran 4 print articles and 4 radio talks. Seventytwo people attended targeted outreach testing: 62 male, 8 female and 2 transgender; 44\% were aged 21-30 years, 20\% had not tested before and $30 \%$ had not tested in the previous 12 months. Thirty-eight doctors and 19 nurses from 5 general practices, a justice health centre and a specialist travel clinic attended 45 -minute workplace education sessions.

Conclusion ACT Testing Month enabled collaboration between government and non-government stakeholders in the sexual health sector to promote HIV, STI and hepatitis testing according to national clinical guidelines. Specific outreach testing was geared to particular at risk groups, whilst workplace-based small group GP education aimed to increase knowledge and reduce 